\title{
Erratum to: Study of Ventilator-Associated Pneumonia in a Pediatric Intensive Care Unit
}

\author{
Pooja Balasubramanian • Milind S. Tullu
}

Published online: 18 December 2014

(C) Dr. K C Chaudhuri Foundation 2014

Erratum to: Indian J Pediatr 81(11):1182-1186

DOI 10.1007/s12098-014-1444-1

The original article contains a mistake in the appendix section. On page 1186, last point in appendix currently reads "Worsening gas exchange [e.g., $\mathrm{O}_{2}$ desaturations (e.g., $\mathrm{PaO}_{2}-$ $\mathrm{FiO}_{2} \leq 240$ ), increased oxygen requirements, or increased ventilation demand]." It should be corrected as "Worsening gas exchange [e.g., $\mathrm{O}_{2}$ desaturations (e.g., $\mathrm{PaO}_{2} / \mathrm{FiO}_{2} \leq 240$ ), increased oxygen requirements, or increased ventilator demand]."

The online version of the original article can be found at http://dx.doi.org/ 10.1007/s12098-014-1444-1.

P. Balasubramanian $\cdot$ M. S. Tullu

Pediatric Intensive Care Unit, Department of Pediatrics, Seth G.S.

Medical College \& KEM Hospital, Mumbai, Maharashtra, India

M. S. Tullu $(\bowtie)$

"Sankalp Siddhi”, Block No. 1, Ground Floor, Kher Nagar, Service

Road, Bandra (East), Mumbai 400051, Maharashtra, India

e-mail: milindtullu@yahoo.com 